\title{
Modes et fonctions du voyage de pèlerinage des Gréco-pontiques en Turquie orientale
}

Forms and Fonctions of the Pontic Coast Greek Inhabitants Pilgrimage Journey to Eastern Turkey

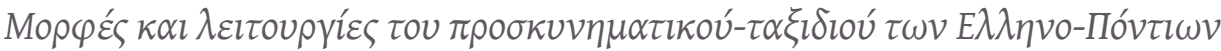

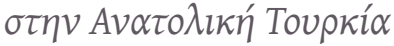

\section{Georges Drettas}

\section{OpenEdition}

\section{Journals}

Édition électronique

URL : https://journals.openedition.org/ceb/3321

DOI : 10.4000/ceb.3321

ISSN : 2261-4184

\section{Éditeur}

INALCO

Édition imprimée

Date de publication : 1 janvier 2004

ISSN : 0290-7402

\section{Référence électronique}

Georges Drettas, « Modes et fonctions du voyage de pèlerinage des Gréco-pontiques en Turquie orientale », Cahiers balkaniques [En ligne], 33 | 2004, mis en ligne le 09 août 2012, consulté le 06 juillet 2021. URL : http://journals.openedition.org/ceb/3321 ; DOI : https://doi.org/10.4000/ceb.3321

Ce document a été généré automatiquement le 6 juillet 2021.

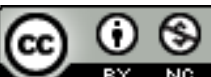

Cahiers balkaniques est mis à disposition selon les termes de la Licence Creative Commons Attribution - Pas d'Utilisation Commerciale 4.0 International. 


\section{Modes et fonctions du voyage de pèlerinage des Gréco-pontiques en Turquie orientale}

Forms and Fonctions of the Pontic Coast Greek Inhabitants Pilgrimage Journey to

Eastern Turkey

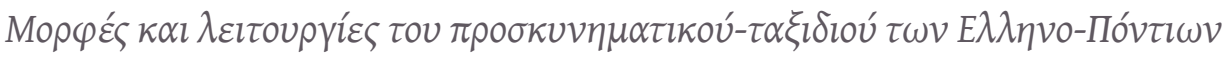

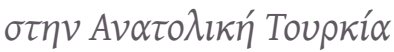

Georges Drettas

\section{Le tourisme de pèlerinage}

1 Pèlerin, du latin peregrinus; le terme spécifique à certaines langues romanes nous renvoie à la genèse d'un phénomène social qui est loin d'être limité aux pratiques ambulatoires que le christianisme occidental a entretenues et favorisées jusqu'aux bouleversements idéologiques que la Réforme protestante, a répandus, de façon directe ou indirecte, sur l'ensemble de l'aire catholique romaine.

2 En fait, le discours ordinaire de la grande majorité des Européens et, par voie de filiation, des Américains, reflète la cristallisation d'une pratique que la modernité n'a pas amoindrie, mais, au contraire qu'elle a rendue plus facile, presque banale. Après Rome, la trop fameuse "Terre sainte ", Saint-Jacques-de-Compostelle, des lieux plus modestes et plus récents n'ont cessé d'apparaître, tels Lourdes, Fatima ou Lisieux. Mais l'on sait bien que l'espace occidental n'est pas premier dans l'histoire du tourisme religieux.

3 L'étude désormais classique de Pierre Maraval (1985) complétée par les travaux des autres byzantinologues (cf. A. Ducellier, 1986) a montré qu'une nouvelle réalité avait émergé à la fin de l'Empire romain unitaire et durant la période proto-byzantine (IV ${ }^{\mathrm{e}}$ $\mathrm{VI}^{\mathrm{e}}$ siècles) dans un espace qui allait être défini comme contenant des «lieux saints" et, de façon plus centrale, la « terre sainte ». Les composantes du processus en question 
sont multiples et si certains éléments sont empruntés au judaïsme prérabbinique, certains traits "postpaïens" ne sont pas à exclure, tel le culte des images ou des reliques. Cela dit, une influence bouddhiste ne peut être exclue, surtout en ce que concerne les espaces liés au monachisme ou à la pratique des acrobaties ascétiques comme celles des stylites.

4 L'Empire chrétien, qui apparaît au IV siècle avec le règne de Constantin, a favorisé la cristallisation d'éléments hétérogènes dont les références sont multiples, qu'il s'agisse des traditions bibliques, puis évangéliques, ou de la vénération des « témoins de la foi ", victimes des grandes persécutions, auxquels vont être associés les " pères du désert ", ermites et thaumaturges. Cet ensemble va constituer un espace sacré dont le centre se situe non pas à Constantinople, capitale de l'Empire orthodoxe, mais à Jérusalem, modèle de la permanence monothéiste.

5 Ce lieu représente dès lors la destination par excellence du voyage sacré, situé au milieu d'un réseau complexe de sites qui lui sont en quelque sorte subordonnés dans une hiérarchie idéelle où les mérites acquis se mesurent à l'intensité symbolique de la distance parcourue. Il convient de souligner le fait que l'espace orthodoxe, anti- ou prochalcédonien, a cristallisé la valeur symbolique de la hiérarchie des lieux après l'expansion de l'islam, aux VIle siècle. Le voyage à Jérusalem et dans ses environs fait acquérir au pèlerin des mérites spécifiques, mais surtout un prestige durable lorsqu'il revient dans sa communauté d'origine.

6 Avec l'islam, la pratique du pèlerinage se voit renforcée à tous les points de vue, puisqu'elle devient une obligation fondamentale, l'un des cinq arkân de la foi. Le terme qui désigne cette réalité, le hajj, en montre la filiation par rapport au judaïsme et au christianisme, puisqu'il est directement apparenté au mot hag qui désigne, en hébreu et en araméen, les grandes fêtes du calendrier, centrées sur le temple de Jérusalem jusqu'à la guerre de 70-71 de notre ère.

7 Lien avec la tradition monothéiste d'une part, mais d'autre part innovation radicale qui s'accomplit avec le changement de qibla, d'orientation de la prière, qui se détourne de Jérusalem pour se fixer vers la Mecque. De la même façon, le pèlerinage, auquel une période spéciale est consacrée, s'effectue à la Mecque, avec un centre matériel très évident, la kaaba, qui représente une forme de litholatrie. Bien entendu, pour les chrétiens de langue arabe, Jérusalem reste la « Sainte », 'al-Quds. Autour de ces centres, des pèlerinages secondaires se développeront, plus ou moins éloignés du lieu modèle. On pense bien sûr aux centres chiites, comme Kerbela, ou à la multiplicité des sanctuaires locaux. Le judaïsme séfarade aura à Safed, en Galilée, une référence locale étayée sur la concentration des maîtres kabbalistes.

8 Au cours du temps, la similarité fonctionnelle du pèlerinage à la Mecque pour les musulmans et à Jérusalem pour les chrétiens orthodoxes apparait de plus en plus clairement, surtout après le $\mathrm{XVI}^{\mathrm{e}}$ siècle, lorsque l'Empire ottoman réunit dans un même ensemble la totalité des territoires sacrés. Cette proximité formelle de la pratique se reflète dans le vocabulaire, puisque les termes arabes hajj "pèlerinage", hajji "pèlerin " passeront en turc hac, hact, et de là dans les autres langues de l'Empire (grec, bulgare, albanais, etc.). Le mérite acquis par le pèlerinage s'exprime par le titre de hacl (en grec $x a d z i(s)$ ) que celui qui a réalisé le voyage à la Mecque ou à Jérusalem a le droit d'ajouter comme préfixe à son nom ou à son patronyme. Aujourd'hui encore, cette possibilité de modifier le nom existe en Grèce (par exemple Kostas devient Xadzikostas, etc.) 
9 Comme nous le laissions entendre au début de notre exposé, les effets matériels du pèlerinage sont aussi importants que ses effets symboliques. En effet, les pèlerins doivent se déplacer sur des distances plus ou moins longues. Ils doivent également disposer de gîtes d'étape où ils pourront se reposer et se nourrir. Enfin, leur sécurité est un élément essentiel au bon fonctionnement du système. On voit aujourd'hui encore que la question des «Lieux saints » demeure un enjeu central des politiques régionales. En osant, à titre d'image, un anachronisme terminologique, nous dirons que les voyages sacrés ont constitué une sorte de prototourisme qui crée un marché des services. Ceuxci pourront être assurés soit par des fondations pieuses, monastères, foyers d'accueil, etc., soit par des professionnels de l'hôtellerie ou de la restauration. On voit aujourd'hui encore, dans la partie occidentale de Jérusalem, l'impressionnant Russian Compound, l'ensemble russe constitué à la fin du XIX siècle pour aider les milliers de pèlerins pauvres qui venaient de toutes les parties de l'Empire russe. Il y a également des installations grecques dans le quartier chrétien de la vieille ville, ainsi que la Greek Colony située à proximité de la route menant à Bethléem, dans la zone appelée aujourd'hui 'emeq rephaïm. Tous les monastères orthodoxes possèdent un foyer, appelé en grec ksenonas, destiné aux voyageurs, mais leurs capacités d'accueil étaient limitées. Avec la modernisation des techniques de transport (bateau, train, puis avion), le temps consacré au voyage s'est considérablement réduit. Parallèlement à ce phénomène, la baisse relative du prix des transports aériens a rendu les pèlerinages accessibles à un grand nombre d'usages potentiels. Cet élément, seul trait dû à la modernité dans la pratique du pèlerinage, a favorisé le développement des services qui se sont inscrits de plus en plus nettement dans ce que les économistes appellent «l'industrie touristique ». Aujourd'hui, les pèlerinages constituent une catégorie particulière des voyages organisés.

Afin de clore cette brève introduction, il n'est pas superflu de résumer la liste des éléments qui permettent de ranger un voyage dans la catégorie " pèlerinage » :

- Il existe un territoire investi d'un caractère sacré. Ce territoire, qui peut se limiter à un endroit précis ou, au contraire, être constitué par une pluralité de lieux, est le but du voyage.

- Le voyageur motive son déplacement par le désir de visiter le territoire sacré.

- La motivation du pèlerinage implique une relation d'identification du voyageur à l'ensemble des traits religieux qui définit le caractère sacré des lieux visités. Il convient de retenir ce point assez fondamental, car il permet de spécifier le pèlerinage au sein de l'activité touristique. Par exemple, si vous n'êtes pas de religion samaritaine, le mont Garizim sera un élément du paysage comme un autre. Si vous ne vous rattachez pas à la tradition juive, la fameuse mer Rouge sera une destination touristique fort appréciée, certes, mais neutre. Enfin, la déambulation du "Chemin de Croix » à partir de la basilique du Saint Sépulcre, dans la vieille ville de Jérusalem, n'a de sens que pour les chrétiens de l'Église latine (les catholiques romains). En effet, les Églises orthodoxes, chalcédoniennes ou monophysites, ignorent cette pratique.

- Le caractère sacré du lieu visité se fonde sur une histoire qui représente un élément essentiel à sa définition, puisque la matérialité spatiale constitue une preuve tangible du récit ou de la tradition religieuse. Pour que cette relation de détermination mutuelle puisse fonctionner, la localisation ne doit pas être mise en doute. La géographie sacrée fonctionne sur le mode de la croyance. Il est clair que la localisation du Golgotha, par exemple, ne doit pas être mise en doute. Dans ce modèle, la situation spatiale garantit la véracité de l'histoire. 
- Du point de vue individuel et collectif, le pèlerin augmente son capital symbolique. Cela dit, le mérite du voyage a fortement diminué avec la généralisation des transports aériens et des fournisseurs de service. Le temps des caravanes qui mettaient plusieurs mois pour atteindre la Mecque ou Jérusalem est bien révolu.

- Si dans la genèse de la pratique, il y a un lien de détermination réciproque entre le tourisme et le voyage de pèlerinage, ce dernier se caractérise par un élément spécifique qui est représenté par la fonction du lieu comme témoin matériel d'une histoire. La référence d'événement doit pouvoir fonctionner comme assise d'identification pour le voyageur, dont l'investissement en temps, en fatigue et en argent se trouve ainsi justifié.

11 C'est ce dernier aspect qui permet de ranger dans la catégorie pèlerinage les voyages des personnes déplacées vers leurs lieux d'origine. Cette activité s'est développée après la Seconde Guerre mondiale et elle concerne plusieurs groupes d'acteurs sociaux. On peut mentionner, bien sûr, les anciens combattants, résistants et déportés qui visitent périodiquement les camps situés en Allemagne, en Pologne ou dans les autres pays occupés (France, Grèce, Ukraine, Biélorussie, etc.). Un autre groupe très important est celui des israélites victimes directes ou indirectes de l'extermination organisée par le Reich. Concernant aussi bien les pays de l'Europe occidentale (France, Italie, Belgique, Allemagne) que de l'Europe orientale (Yougoslavie, Grèce, URSS, etc.), les populations survivantes se rendent périodiquement en Pologne, dans les camps d'extermination, dont le plus connu est celui de Auschwitz-Birkenau.

On remarque un processus de pérennisation de ces voyages, qui peuvent être accomplis aujourd'hui par des petits-enfants, voire des arrière-petits-enfants des victimes. L'histoire familiale définitoire de chaque individu constitue un élément central du pèlerinage. Parallèlement au vécu familial, la visite des camps, des cimetières ou de ce qui reste des communautés disparues, revêt un contenu religieux, même chez les incroyants. En effet, la mémorisation est une notion centrale du judaïsme, et l'évocation des défunts relève du respect que l'on doit rendre à l'être humain en général. Bien entendu, la Shoah représente une donnée historique spécifique, mais certains de ses effets se rencontrent dans des situations moins tragiques.

On remarquera que la mode actuelle de la «mémoire » dans les sciences sociales a été en partie déterminée par les débats qui ont vu le jour lorsque les courants négationnistes, présents aux USA et en Europe, ont mis en question la réalité du souvenir oral, du témoignage individuel qui n'est pas matérialisé dans un document écrit. Cette problématique est très importante d'un point de vue méthodologique. On doit constater en effet que le document écrit dépend de l'oral dont il ne reflète parfois qu'une falsification parfois grossière. Les autorités allemandes de l'Europe occupée ont illustré abondamment ce genre de pratique, à tel point que les révisionnistes avaient beau jeu de réfuter la véracité du matériel produit par l'historiographie traditionnelle. Dans ce cadre, où le témoignage oral se voit dénier toute possibilité de servir de moyens de preuve, n'importe quel événement se voit mis en doute, dans la mesure où des évidences criantes ne relèvent plus que du domaine de la doxa.

Dans ce relativisme délirant, toute ébauche d'histoire sociale est assimilée au roman familial, dont la psychanalyse freudienne a montré l'importance. Or, de façon presque paradoxale, le lieu supplée au discours face aux mises en doute les plus radicales, car s'il est nommé, il s'est forcément passé quelque chose. Nous retrouvons par là un motif ancien que l'on repère dans la genèse de tous les pèlerinages où l'histoire et la croyance se confondent, car la fonction principale du repère local est ailleurs. Un exemple simple 
permettra d'illustrer cette question: Saint Georges, dont le culte est excessivement important dans toutes les régions du Proche-Orient, des Balkans et du Caucase, s'est vu attribuer au moins deux tombeaux. L'un se situe à Lidda/Lod, en Israël, l'autre se trouve quelque part en Cappadoce. Dans ce cas, la localisation précise, le sanctuaire, ne constitue pas un élément fondamental du culte. Le saint, du reste, se caractérise par une mobilité remarquable. Il a pu être ainsi adopté et adapté dans les environnements les plus divers.

\section{Introduction au voyage} personnes déplacées à la suite d'un événement historique majeur qui constitue une rupture radicale et douloureuse. Dans ces cas, l'histoire du groupe se confond avec les histoires familiales et individuelles.

\section{des pertes humaines considérables ainsi que des déplacements de population d'une} ampleur inconnue jusque-là, en Europe orientale et au Proche-Orient. terminer la guerre gréco-turque, conclue par le traité de Lausanne. Il faut bien comprendre ici un fait essentiel : l'organisation internationale des États, la Société des Nations, visait, comme aujourd'hui l'ONU, à réguler les conflits régionaux en créant des mécanismes juridiques reconnus par tous. Or, dans les années 20, l'échange de populations était proposé par nombre d'experts comme le seul moyen de régler efficacement des problèmes ethniques qui avaient mené à la guerre.

18 Le fait que le déplacement obligatoire puisse constituer un drame très profond pour les populations concernées n'est presque jamais évoqué dans la littérature spécialisée de l'époque. Or, la douleur du déplacement, appelé souvent exode, peut devenir une des composantes de la mémoire du groupe. Mais l'indifférence des experts internationaux de l'époque concerne également le fait que l'échange des populations s'applique à deux groupes humains dont les détresses se répondent. Rappelons que sur ce point, l'excellente Encyclopédie ottomane (Tanzimat'tan ... ) a consacré un exposé de fond à la question des göçmen, qui se pose tout au long du XIX et au début du XXe siècle.

19 Le nombre de personnes échangées, du côté grec, se monte à 1250000 , auxquels s'ajouteront des réfugiés non recensés ainsi que des Grecs du Caucase russe, en particulier des territoires de Kars et Ardahan, qui ont été rétrocédés à la Turquie, en 1921. Il faut préciser que la complexité de certains flux migratoires durant la guerre nous oblige à considérer les chiffres des institutions patronnées par la SDN comme relatifs.

Dans cette masse globale, le nombre des pontiques grecs orthodoxes restés en Turquie entre 1914 et 1923 s'élève à 200000 personnes environ. Ce groupe sera donc transféré en Grèce, principalement en Macédoine et en Thrace occidentale, en rejoignant les Pontiques réfugiés de l'Empire russe, qui est en train de devenir l'URSS. Il s'agit donc d'une masse démographique considérable pour l'époque, qu'on la mette en rapport avec la population totale de l'État ottoman en Anatolie ou avec celle du Royaume de Grèce. 
21 La population pontique a été installée majoritairement dans des habitats ruraux ou semi ruraux. En règle générale, les familles sont restées groupées selon leur village d'origine, au Pont (v. G. Drettas, 1997). On pourrait penser que ce mode d'installation par regroupement villageois ou familial a favorisé le maintien d'une culture d'origine, comme c'est le cas chez les Caucasiens de Turquie (Ubux, Adyghés, Abkhaz, Tchétchènes, Géorgiens adjars, etc.). Les cas évoqués posent évidemment une question difficile à l'anthropologie des migrations et que l'on pourrait formuler comme suit : certaines cultures favorisent-elles la cristallisation d'une base historique du groupe en dépit de la rupture avec le milieu originel ? Il n'est pas question de traiter ce thème complexe, dans le cadre limité de notre étude, mais nous remarquerons que nous avons à faire, dans nos exemples, à des cultures caucasiennes ou péricaucasiennes. Celles-ci, comme les Adygués, par exemple, se sont maintenues, en Turquie, après l'exode de 1864.

Chez les Pontiques de Grèce, on observe un déséquilibre dans les modes d'adaptation à la territorialité, qui se reflète dans la production d'un certain nombre de stéréotypes, présents dans le discours ordinaire. Je ne mentionnerai ici que les plus importants.

On remarque ainsi la tendance à exagérer le nombre réel des Grecs pontiques vivant en Grèce. Popularisée par les notables et les lettrés, ce que l'on pourrait appeler une démographie imaginaire a au pour effet de brouiller des questions fort importantes, comme l'histoire sanitaire (mortalité infantile, paludisme, reprise démographique dès la fin des années 40 semble indéniable, mais son intensité demeure hypothétique. Avec l'urbanisation due à la guerre civile, la pratique, limitée certes, des mariages mixtes, la définition identitaire peut faire problème. Compte tenu de l'idéologie patrilinéaire commune à l'ensemble des populations de nos régions, la ponticité paternelle est suffisante pour assurer celle de ses enfants, mâles et femelles. Nous devons retenir cet élément qui peut intervenir dans les effets du voyage au Pont chez certains individus. On constate en fait qu'il y a aujourd'hui deux degrés de ponticité. Le premier degré est celui qu'assument les personnes dont les deux parents sont pontiques et le second degré celui des enfants issus des couples mixtes.

On observe également ${ }^{1}$ un clivage idéel entre la patridha, le pays du père (ou du grandpère), là-bas, en Turquie, et l'ici, le territoire d'accueil, en Grèce. Le pays d'origine se voit attribuer toutes les qualités, du sol, du climat, de l'eau et de la saveur des fruits. La terre d'accueil est fortement dévalorisée, à tous les points de vue, et cela aussi bien chez les réfugiés ruraux que chez les réfugiés urbains. Dans le jeu du positif perdu à jamais et du négatif qui relève de la contrainte, les histoires familiales se fondent sur une sélection des souvenirs transmis. C'est-à-dire que les éléments objectivement négatifs de la société pontique sont parfois oubliés, parfois simplement occultés.

Dans le sentiment de négativité, les conflits réels ou symboliques avec les autochtones (en grec vтó 101 ) occupent une place centrale qui renvoie à une thématique fréquente du discours des réfugiés. En théorie, le déplacement correspondait à un échange, mais en réalité le territoire d'accueil n'était pas vide. Les premiers habitants ont ressenti des sentiments d'injustice et de frustration lorsqu'ils ont dû partager avec des intrus un territoire aux dimensions modestes. La pratique de la colonisation que toutes les instances internationales préconisaient a eu pour effet de déclencher dans les populations autochtones une attitude de rejet profonde et durable vis-à-vis de décisions étatiques qu'ils ont assimilées à des spoliations. 

moniales. Il est géré par un certain nombre d'employés de l'Association Panaghia Sumela, ainsi que par un prêtre qui vit à proximité de l'ensemble avec une famille nombreuse. Traité comme «lieu de mémoire» dans la littérature consacrée aux Pontiques de Grèce (cf. Bruneau, 1998), la Panaghia Sumela a connu un développement particulier qui l'a constituée en marqueur de la ponticité explicite dans la trajectoire historique qui va du pouvoir absolu de la droite monarchiste, après 1949, à la dictature des colonels et à l'arrivée de la gauche aux affaires, en 1981.

Le balancement entre ces deux types de territoire, dont le premier est perdu et dont l'autre est imposé ou partagé de force, a favorisé le développement de ce que l'on pourrait appeler une "conscience spatiale malheureuse", intense chez les réfugiés d'Asie Mineure, mais présente également chez les autochtones. Le lien négatif au territoire quotidien a facilité probablement l'exode rural et l'émigration massive vers l'Europe occidentale (R.F.A., Belgique, France) après la Seconde Guerre mondiale. essentiellement sur les réseaux familiaux partageant une référence commune à la micro-région d'origine. Ces solidarités ont été mises à l'épreuve pendant l'occupation et la guerre civile qui a suivi (1943-1949). Les clivages politiques et sociaux, communs à la situation nationale et aux situations régionales, ont été plus importants que les clivages ethniques. On peut dire aujourd'hui, sans exagérer, que l'EAM-ELAS (Front national de libération / Armée nationale populaire de libération) auquel succède, en 1946, l'Armée démocratique a constitué un creuset d'intégration nationale.

La dynamique efficiente qui avait vu le jour durant la lutte contre les Allemands et les Bulgares a été brutalement interrompue par la guerre civile. Celle-ci s'est déroulée principalement en Grèce du Nord et a par conséquent touché intensément les populations pontiques.

Sorti vainqueur de la guerre civile le pouvoir de la monarchie des Glüksburg, soutenu par les USA et l'OTAN, a favorisé les initiatives visant à récupérer les Pontiques ruraux. C'est ainsi que l'Association pontique de Salonique "Panaghia Sumela" réussissait à fonder, en 1951, un monastère du même nom. Le lieu où se situe l'ensemble monastique se trouve sur les pentes du Vermion, à quelques kilomètres de Verria, en Macédoine centrale. Le cadre montagneux est impressionnant et le choix de cet espace a été déterminé par sa ressemblance physique avec la cordillère pontique d'où étaient originaires les responsables de l'Association.

Au départ, une petite église contenait une icône de la Vierge, fort abîmée, mais provenant justement des objets ramenés du monastère pontique de Sumela. L'ensemble a été tout de suite conçu comme un lieu de pèlerinage national. À l'époque de la dictature militaire (1967-1974) qui mettait en avant le thème de la «Grèce de Grecs chrétiens ", toutes les institutions religieuses ont été fortement subventionnées ${ }^{2}$. Dans ce cadre, le site s'est enrichi de toute une série de constructions propres à loger les pèlerins, en particulier les originaires de telle ou telle région du Pont.

Comme c'est souvent le cas en Grèce, le monastère est en réalité vide de moines ou de Afin de mieux comprendre l'investissement symbolique dont fait l'objet le " pèlerinage interne » sur le site de Sumela, il faut exposer brièvement les dimensions idéologiques de ce qu'il conviendrait d'appeler le complexe pontique, dans la Grèce des cinquante dernières années. C'est-à-dire que l'on observe un clivage complémentaire au sein des discours produits par l'intelligentsia pontique. 

d'autre part. Ce mouvement où nombre de symboles étaient exhibés signifiait aussi que la dichotomie entre une droite radicale et une gauche communiste vouée à l'héroïsme du désespoir était désormais dépassée au profit de configurations complexes. Les deux Congrès mondiaux des Pontiques, de juillet 1985 et de juillet-août 1988 à Salonique, ont exprimé les différents aspects de la réalité pontique contemporaine. Or, celle-ci a été déterminée par l'expérience nouvelle qui constituait le retour aux origines. 


\section{Le retour au pays}

sud et est-ouest, vers le Caucase et l'Iran. Les voyages en ces régions est attestée depuis fort longtemps et l'on évoquera, à l'époque ottomane, J. B. Chardin, J. Pitton de Tournefort, Evliya Çelebi, plus récemment J. P. Fallmerayer, enfin, au début du $\mathrm{XX}^{\mathrm{e}}$ siècle, le grand linguiste géorgien Nikolaj Jakovlevitsh Marr, le philologue anglais Douglas Dawkins, le géographe Stratil-Sauer, pour finir avec les archéologues britanniques Anthony Bryer et David Windfield. Au début du $\mathrm{XX}^{\mathrm{e}}$ siècle, certains voyageurs grecs ont également visité le Pont, par exemple, Papamichalopoulos, qui a publié un compte-rendu de son parcours en 1903, à Athènes. Vers 1960-1965 des voyages au Pont commencent à être effectués par des notables (fonctionnaires divers, politiciens, anciens militaires, etc.) dont la plupart étaient nés en Turquie ou, pour certains d'entre eux, au Caucase russe. Ces déplacements ne concernent cependant que très peu de monde. Il convient de les mentionner, car des comptes rendus plus ou moins précis en sont parus dans les publications destinées aux populations pontiques. Ces publications sont de trois sortes :

- Périodiques, dont le plus connu est sans conteste le Pontiaki Estia, qui, fondé en 1950, existe encore aujourd'hui ;

- Almanachs divers publiés à l'occasion d'un anniversaire par telle ou telle association (comme les originaires de Krom, à Kalamaria) ;

- Des plaquettes plus ou moins importantes, dont le modèle est l'itinéraire au Pont, bref récit de voyage qui associe la description des lieux à la vulgate historique constituée par les éléments que le climat politique grec tolère.

Dans la construction de ce discours standard, les ressources de la recherche scientifique, qu'il s'agisse de la byzantinologie ou de la turcologie, sont très peu sollicitées. On relèvera quand même deux éléments récurrents qui figurent dans la plupart des récits :

- tout d'abord, les voyageurs racontent, non sans émotion, la rencontre, dans la population musulmane locale, de personnes âgées qui ont connu la période d'avant 1924 et, dans le meilleur des cas, la maison paternelle, parfois même natale. Il s'agit alors de la constitution d'une évidence archéologique, le bâtiment fonctionnant comme une métonymie généalogique, une sorte de monument historique individuel.

- Le deuxième élément est négatif. Dans la vulgate de l'époque, les Pontiques sont présentés comme des chrétiens exemplaires doués d'un degré maximal de grécité. Quant aux Pontiques musulmans, ils sont totalement occultés.

Aussi limitée soit-elle, la pratique de ces voyages a eu un écho dans la population pontique. Il est difficile, voire impossible, de mesurer l'impact de cette connaissance 
diffuse de la réalité originelle, mais il est certain qu'elle a facilité la vogue touristique qui caractérise la phase postérieure à 1974 .

L'organisation des voyages de groupe est assumée, en Grèce, par ce qu'on appellerait communément des PME. En Macédoine, nombre de ces entreprises sont pontiques. Elles ont toutes profité du développement touristique de la Turquie dans les années 70, et de l'ouverture du marché grec au tourisme indigène facilité par le coût de la vie turque ainsi que par les taux de change qui favorisaient les Grecs.

Dans un premier temps, on a vu se multiplier les voyages dans ce que l'on pourrait appeler la Turquie proche, c'est-à-dire vers Istanbul, Izmir ou Bursa. Ces voyages sont devenus extrêmement fréquents en dépit de la tension qui se manifeste entre les deux pays non pas tant à cause du problème chypriote qu'à cause du plateau continental de la mer Égée. À cette situation de tension récurrente correspond une très nette volonté de réviser l'histoire officielle pour la majorité des intellectuels progressistes, qu'ils aient participé ou non à la résistance contre la dictature. L'historiographie de droite, issue de la guerre civile, se verra marginalisée avec le nationalisme qu'elle servait à fonder. L'histoire critique inspirée par les principes du matérialisme dialectique accorde une attention soutenue aux "racines» orientales de la nation grecque contemporaine, et bientôt le voyage dans la Turquie proche est devenu assez banal. Certains se hasardent à visiter le Plateau central et la Cappadoce.

Le retour aux sources alimente quelques-uns des thèmes typiques de ce qu'il convient d'appeler le néo-nationalisme de gauche. Ce dernier est, certes, un échafaudage complexe dont l'une des caractéristiques est de réintégrer la dimension anatolienne de la "grécité » actuelle 6 . Constitué d'un ensemble de représentations contradictoires, le néo-nationalisme des années 80 allait être mis à rude épreuve par deux phénomènes concomitants, les guerres menées par les Serbes dans les Balkans ainsi que l'intégration renforcée de l'État grec dans une Union Européenne élargie.

Comme nous le précisions plus haut, le voyage au Pont, dans la région appelée en turc Karadeniz ( mer Noire) et Lazistan, revêt une importance toute particulière en raison du poids démographique de la population pontique dans l'ensemble grec et surtout en Grèce du Nord, Macédoine et Thrace. Ce poids a eu pour conséquence la constitution d'un monopole de fait, dans la mesure où la très grande majorité des agences de voyages qui propose les séjours au Pont sont gérées par des Pontiques. Nous nous trouvons dans le cas de figure du pèlerinage lorsque le client est lui-même pontique, soit né en Turquie, soit né en Grèce des parents immigrés en 1924.

Je relève ce détail qui me paraît très important, parce que la transaction commerciale implique une connivence toute particulière entre le prestataire de services et son client. En fait, le vendeur peut jouer sur la fibre identitaire pour fournir des services de qualité médiocre sans risquer de trop gros problèmes avec ses clients. En tout état de cause, le pèlerinage justifie quelques fatigues, et le gain symbolique est toujours assuré. Aujourd'hui, tout groupe pontique se divise entre ceux qui ont été au Pont, au moins une fois, et ceux qui demeurent ignorants du pays. Il faut préciser quand même que le développement du tourisme grec s'appuie sur un phénomène similaire en Turquie, au point qu'il y a quelques années, des posters du monastère de Sumela étaient collés dans le métro parisien.

Il y a fondamentalement deux groupes de touristes pèlerins. Le premier est constitué de gens qui connaissent leurs origines pontiques tout en ignorant le lieu d'origine précis 
des parents. Dans ce cas, Trébizonde fonctionne comme une sorte de toponymie générique. Ce groupe appréhende la région pontique dans une globalité relativement indifférenciée et il peut se contenter d'une information minimale. L'autre groupe comprend tous ceux qui connaissent très précisément la microrégion d'origine de leurs parents. Ils ont une représentation plus réaliste du pays pontique et ils ont pour but de revoir l'habitat d'origine, autant que faire se peut. Ces clients sont, par conséquent, plus exigeants que ceux du premier groupe. On pourrait parler ainsi de deux modalités du voyage, celle du pèlerinage général et celle du pèlerinage spécifique qui se fonde sur une mémoire familiale bien définie.

Les itinéraires sont peu variés. Il y a la possibilité de se rendre par avion d'Athènes ou de Salonique à Istanbul et/ou à Trébizonde, et de là on peut rejoindre un circuit local. Mais le trajet le plus classique se réalise en autocar, au départ de Salonique, et il comprend les étapes suivantes: Istanbul-Bolu, où l'on pénètre dans la Turquie profonde, Ankara, Çorum, Merzifon et Samsun, qui marque le début de la région pontique proprement dite. La route côtière qui relie Samsun à Trébizonde permet de réaliser l'importance géographique du pays pontique sur une distance de $371 \mathrm{~km}$. Le tracé routier traverse tous les habitats littoraux situés entre Samsun, capitale du Pont occidental, et Trébizonde, centre du Pont oriental, soit, dans l'ordre : Çarşamba, Terme, Ünye, Ordu (en grec Kotyora), Bulancak (Pulançak), Giresun (Kerasunta), Tirebolu (Tripoli). À l'est de Trébizonde, la route passe par Sürmene et of pour atteindre le Lazistan à Rize. Cette région qui correspond au littoral de l'ancienne Lazique ( $\Lambda \alpha \zeta$ \ки́, chez Procope) est depuis fort longtemps musulmane en totalité et elle ne présente qu'un intérêt historique, non familial, pour le pèlerin.

50 La montagne pontique, là où se situait la majorité des habitats chrétiens, est accessible à partir de Trébizonde. La ville dispose d'une bonne infrastructure hôtelière et de nombreux taxis. Elle sert donc de centre pour des excursions en montagne. Il s'agit en effet, pour les plus motivés de nos pèlerins, de visiter les habitats qui se trouvent à droite et à gauche de la route qui relie Trébizonde à Gümüşhane, sur une distance de $90 \mathrm{~km}$ environ.

Les deux étages du versant montagneux, orienté nord-sud par rapport à la mer, peuvent ainsi être parcourus. D'abord, la première tranche située en dessous de la ligne de 2000 mètres, avec la région de Maçka (Matsuka) et, bien entendu, le monastère de Sumela. La deuxième tranche, qui se situe en dessous des sommets culminant à 3000 mètres environ, constitue le territoire de la Xaldhia médiévale et comprend les microrégions de Krom (Kurum-dere), de Torul (Ardhasa), de Gümüşhane (Kan) et de Santa. Dès que l'on pénètre dans ces zones le long des fleuves étroits qui surgissent des pentes, on peut faire l'expérience du caractère alpin que présente le paysage pontique. Dans la première zone ou région, ou région de Maçka, les voyageurs vont visiter le monastère dédié à la Vierge de Sumela, qui, en dépit de son état de ruine, constitue un site tout à fait impressionnant. Cette visite permet de vérifier l'authenticité des récits de vie. On peut signaler le fait que certains groupes emmènent avec eux des enregistrements religieux afin de pratiquer des rituels de commémoration dans l'une des chapelles abandonnées du monastère. On observe une assez grande tolérance des autorités locales face à ces pratiques. La deuxième zone, située en gros au-dessus du fameux col de Zighana (2000 m d'altitude), s'étend des deux côtés de la route qui, par Torul, Gümüşhane, Pirahmet et Bayburt, se dirige vers Erzurum. 

retour est fort complexe. Le désir de retour repose sur une tension entre l'imaginaire et une réalité qui ne se manifeste tout d'abord que comme une possibilité. Le domaine de l'imaginaire est peuplé de fantasmes qui favorisent des identifications plus ou moins élaborées à des représentations labiles. Celles-ci finissent souvent par se cristalliser sous la forme de stéréotypes et d'idées reçues qui parsèment le discours ordinaire de la ponticité. toute notion de "retour ", mais également les êtres qui peuplent cet espace retrouvé, car il ne s'agit pas d'un désert. La rencontre avec la réalité humaine du territoire des origines alimente une thématique conversationnelle qui s'organise autour de quelques éléments saillants.

La présence de la masse turque ne pose pas de problème particulier puisqu'elle correspond à la dichotomie classique: Grecs versus Turcs. L'existence de ponticophones musulmans bouleverse nombre d'idées reçues dans le discours habituel des Pontiques de Grèce, qui assimilent fréquemment la caractérisation ethnique et le groupe confessionnel. L'étayage religieux de l'identité 9 se heurte aux signes visibles de l'islam pratiquant: femmes recouvertes d'un grand voile de type çarşaf, dans les 
marchés, vieillards portant un turban, réalisé souvent avec un cache-col, nombreuses mosquées en construction, etc.

Le fort attachement des populations de la Turquie du Nord-Est à l'islam, qu'il s'agisse des Çepni, des Turkmènes, des Pontiques ou des Lazes, commence à faire l'objet d'observations sociologiques et anthropologiques (cf. M. Meeker, 2002). Cela gêne considérablement les Gréco-Pontiques qui reviennent au pays.

Le thème du clivage religieux, déterminant dans l'histoire du groupe, alimente les conversations. Il s'agit en réalité d'une différence sensible, objective, qui s'oppose à toutes les similarités relevées dans l'univers quotidien. De ce point de vue, essentiel dans les contacts avec la population locale, celle-ci fournit une mesure de l'authenticité, de la présence d'éléments du passé récent, dont certains n'étaient connus que par ouï-dire.

61 Le domaine culinaire propose des realia qui représentent la culture villageoise traditionnelle. Ainsi l'on trouve encore les pujinte, les lavajo, le xavits, mais surtout les fameuses kintéas, mets à base d'orties cuisinées. Cette préparation, qui a disparu de la diète pratiquée en Grèce, représente dans le discours ordinaire une métonymie culinaire de la ponticité plus déterminante que les champignons (en pontique : takukuvakc) ou les escargots (en pontique: ta- koxlidœ), que les Grecs balkaniques et insulaires ne consomment pas ${ }^{10}$.

Les mets traditionnels représentent une "culture du beurre " similaire à celle du Caucase méridional. Cet ensemble se fonde sur l'association du beurre et du yaourt aux céréales, crues ou torréfiées. Le littoral fournissait à la montagne les fameux xampsia, et la viande, rare, était normalement cuite dans sa graisse et séchée (c'est le kavurma). En Grèce, après la Seconde Guerre mondiale, l'huile d'olive a pénétré dans les plus petits villages et a remplacé le beurre dans la plupart des préparations culinaires. Les plats traditionnels que l'on retrouve en Turquie servent à mesurer une certaine distance chronologique. Il s'agit au fond de la mise en relation du présent avec un avant que les Pontiques musulmans proposent d'une façon normative. Ce sont eux qui ont encore la maitrise des pratiques culinaires qui ne sont pas oubliées, mais tombées en désuétude. Les voyageurs réapprennent quelque chose qu'ils ne connaissaient plus, ou ils reviennent à un mode de vie rural que certains d'entre eux avaient connu dans leur enfance.

Il convient de préciser que chaque ensemble mémoriel est fonction des générations en présence. Le domaine du souvenir n'est pas homogène. Cela est manifeste dans l'élément sensible que constitue le costume féminin, porté par les femmes des villages et que l'on peut voir sur les marchés urbains. Ce costume, dont la pièce la plus visible est le laxor ou jupe-tablier recouvrant le pantalon, salvar, a été stylisé en Grèce pour être porté par les jeunes filles des groupes de danse pontique. Les jeunes pontiques, qui n'ont jamais vu le vêtement porté dans la réalité du quotidien, par leurs grands-mères, vont percevoir son existence comme un trait exotique, parmi d'autres. Pour les personnes âgées (tranche d'âge de 70-85 ans), la vision de ces femmes musulmanes en costume, souvent courbées sous le yelék "fardeau», les ramène aux souvenirs de l'enfance.

64 La tradition musicale joue un rôle important dans les interactions produites par le voyage. En Grèce, les chansons et les danses sont, sans conteste, l'élément de la culture pontique le mieux conservé, même si l'ensemble a subi une certaine folklorisation. Certains groupes emmènent avec eux un joueur de kemendjé, lequel est chargé d'animer 
la route, si longue. Ce personnage, traditionnellement respecté et choyé par ses auditeurs, établit un contact rapide avec la population locale. On amène, si possible, un joueur de kemendjé indigène, et l'on évalue les différences entre le style de jeu, la façon de danser ou le répertoire textuel ${ }^{11}$.

Quant aux groupes folkloriques locaux, ils portent le costume laze, comme en Grèce, en particulier la fameuse zipka, et ils exécutent les mêmes danses que les Gréco-Pontiques, seulement sur un tempo plus rapide. Ils exhibent des armes et des insignes nationalistes ${ }^{12}$. La confrontation des images réunit des supports d'identifications et des indices de différences qui renvoient toutes au clivage fondateur de la culture pontique et à la recherche d'une normativité catégorielle permettant de ranger les éléments authentiques dans les replis d'une mémoire dispersée.

La question des normes apparaît très nettement avec la pratique langagière. En effet, il est excessivement rare que les Pontiques nés en Grèce aient une connaissance du turc. La seule langue de communication possible est le pontique, avec les musulmans ponticophones. Les échanges langagiers amènent à considérer que la variété ${ }^{13}$ parlée par les musulmans est plus archaïque, plus riche et plus « correcte » que le pontique des chrétiens. Là encore, le pays pontique reste un réservoir de l'authenticité des pratiques traditionnelles.

67 Reprenant ma remarque initiale, je soulignerai le fait que l'authenticité de la culture dite traditionnelle est aujourd'hui détenue par la population musulmane, ce qui modifie forcément le rapport au territoire imaginaire des Pontiques de Grèce ${ }^{14}$. Nombre de faits viendraient corroborer ce constat, mais je me contenterai d'évoquer un exemple qui me semble illustrer parfaitement cette question.

À partir du 18 août a lieu un grand rassemblement festif sur le yayla ou parxar de Haçka, situé au-dessus de Tonya, à 2000 mètres d'altitude environ. Cette fête, appelée panoïr en pontique, est dédiée aux saints missionnaires qui convertirent les populations locales à l'islam, au XVII ${ }^{\mathrm{e}}$ siècle. Au-delà des türbe, une foule de marchands divers, de pèlerins et de visiteurs venus des régions voisines se rassemble pour flâner, manger, danser et même pratiquer des démonstrations équestres. Dans ce genre de rencontre, on peut voir un concentré des éléments de la culture pontique. Pour les chrétiens qui observent cette fête, la résonance affective est particulière, puisque la ponticité réelle est associée au triomphe de l'islam, alors que les grands monastères dédiés à la Vierge ou à saint Georges ne sont plus que ruines. Cette fête, qui représente aussi un pèlerinage annuel au niveau régional, propose les images d'une ponticité parallèle, d'une autre histoire d'où surgissent des alliés, voire des héros, du mouvement kémaliste, et cela dès 1919.

\section{Leçons de choses}

Il y a des histoires dramatiques qui peuvent être acceptées sans qu'il faille les occulter pour autant.

Comme tout voyage, le retour au Pont se nourrit d'un certain nombre de visites et de visions. Au-delà de ce marché d'impressions qui fait partie intégrante de l'activité touristique moderne, on observe une prise de conscience déterminée par l'observation de la réalité quotidienne. Cette prise de conscience est celle d'une rupture qui s'exprime à travers plusieurs thèmes conversationnels. 
71 L'image stéréotypée du Pontique chrétien manifestant une sorte d'hypergrécité exhibitionniste se heurte à la réalité de ces Pontiques musulmans qui manifestent les signes d'un nationalisme turc exprimé selon un mode kémaliste encore intact (v. Meeker, 2002).

72 La comparaison systématique des prix courants et des niveaux de vie fait pencher la balance en faveur de la Grèce, où, somme toute, l'on vit mieux qu'en Turquie. Plusieurs analystes spontanés formulent la comparaison dans les termes d'une échelle chronologique relative : du point de vue du mode de vie et du niveau économique, la Turquie pontique en est au stade où était la Grèce (i.e. la Macédoine) il y a quarante ans.

73 À cette évaluation positive en faveur de la Grèce correspond le constat d'une rupture inéluctable. Le pèlerinage permet à certains de vérifier l'ancrage territorial du discours familial, mais il ne soutient pas un désir de retour au sens d'une réinstallation, sous quelque forme que ce soit, au village ancestral ou à la ville.

Le conflit qui est à l'origine du déplacement est inscrit en mémoire de façon confuse, mais très profonde, et son caractère d'événement total, irréversible, apparaît dans les allusions discursives et les non-dits. On remarque que le sentiment très profond de coupure historique ne produit pas de rancœur exprimée ou de revendication irrédentiste, comme on a pu l'observer dans d'autres cas de déplacement forcé. Il y a, bien sûr, des exceptions à ce constat, mais elles demeurent marginales. On observe, en fait, une dynamique qui opère sur la relation entre la représentation du territoire comme garantie de l'origine individuelle et collective, et le domaine des lieux de la vie quotidienne, qui relève de la rationalité économique et sociale.

La «leçon de réalisme » qu'offre le voyage peut être mise en lumière, par effet de contraste, avec l'admiration sincère que les gens simples, les Pontiques moyens, éprouvent envers les comportements violents attribués aux musulmans locaux. Ainsi, le port d'armes, illégal, l'organisation en familles politiques, la pratique des meurtres et de la vendetta, la transgression de la légalité républicaine, sont positivement commentés comme autant de traits typiques, mais il n'est jamais question de les adopter à nouveau. Il est imprudent de conclure au sujet d'un processus qui continue d'évoluer de façon parfois rapide. Mais, comme je le signalais au début de cet examen, le pèlerinage doit offrir quelque chose à celui qui le pratique, et dans notre cas, je pense qu'il nous invite à formuler une question d'ordre général: dans les tourments territoriaux produits par les États-nations contemporains, l'attachement à deux territoires, l'un d'appartenance nationale et de résidence, l'autre de droit symbolique et de parcours provisoire, n'apporte-t-il pas un remède aux effets incurables du drame que les personnes déplacées ont vécu?

Dans le cas pontique, on observe une modification des réseaux de l'imaginaire dans le sens du principe de réalité qui permet de définir la réalité du manque associé à une légitime nostalgie. Le droit au retour dans l'ordre symbolique permet d'éviter les délires irrédentistes. Je dirais que la vision du pays réel tempère l'image identificatoire qu'il construit. 


\section{BIBLIOGRAPHIE}

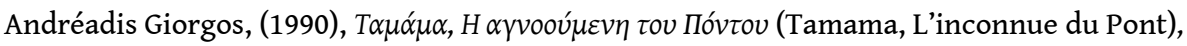
Salonique : Gordios, 1990. Publié aussi en langue pontique.

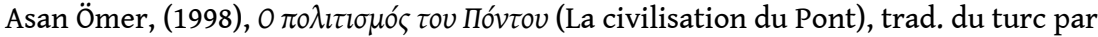

A. Fotopoulou, Salonique : Kyriakidis.

Beller-Hahn, Ildiko \& Hann Chris, (2001), Turkish region. State Market \& social identities on the East Black Sea Coast, Santa Fe: School of American Research Press.

Drettas Georges, (1997), Aspects pontiques, Paris : ARP.

Drettas Georges, (2003), « Diaspora et migration éléments pour une histoire linguistique de l'ensemble grec », Diasporas/Histoires et Sociétés n² 2, Toulouse, pp. 36-54.

Drettas Georges, (1997), « Propos sur l'entretien d'une mémoire importune. Récit de Tona, la femme du prêtre (1914-1924) », CLO n 42, Récits de vie 2, pp. 73-105.

Ducelier Armand, (1986), Byzance et le monde orthodoxe, Armand Colin, Paris, 1986.

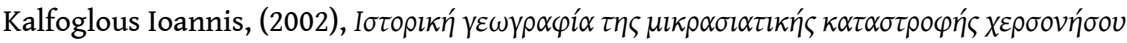
(Géographie historique de la péninsule micrasiatique), trad. Stavros Anestidis, Athènes : KMS.

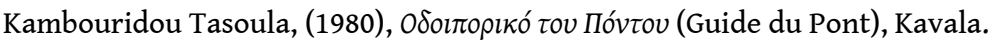

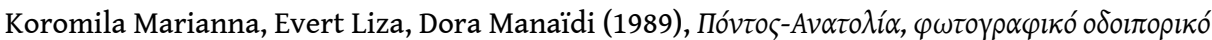
(Pont-Anatolie, guide photographique), Athènes : L. Bratsioti.

Maraval Pierre, (1985), Lieux saints et pèlerinages d'Orient, Cerf, Paris.

Meeker Michael, (2002), A Nation of Empire. The Ottoman Legacy of Turkish Modernity, Berkeley: University of California Press.

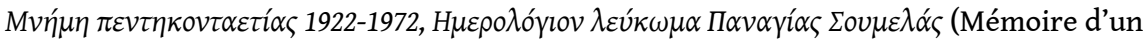
cinquantenaire 1922-1972, Journal de Panaghia Sumela), (1973) Salonique.

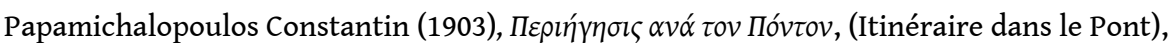
Athènes.

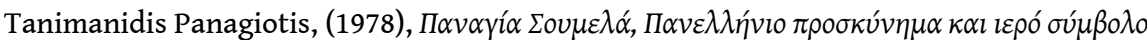
(Panaghia Sumela, pèlerinage panhellénique et symbole sacré), Salonique : Association Panaghia Sumela.

\section{NOTES}

1. Il faut bien préciser qu'il s'agit toujours de quantités relatives. On est obligé de tenir compte des témoignages individuels ou familiaux, mais on ne peut généraliser sans prudence ce qui relève de la doxa. En effet, la totalité pontique n'est pas observable.

2. La Grèce n'est pas un pays laïc, et il s'agit bien sûr de sommes qui venaient s'ajouter au budget courant.

3. Il n'est pas sans intérêt de remarquer que beaucoup de ces notables (politiciens, professeurs du secondaire, médecins, administrateurs, commerçants, etc.) étaient originaires de familles qui 
avaient émigré vers la Russie. Certains chefs des bandes armées collaboratrices pendant la guerre étaient d'anciens officiers de l'armée impériale russe.

4. Le Parti communiste grec a été fondé peu de temps avant l'arrivée des réfugiés d'Asie Mineure. Il a été interdit de 1936 (dictature du général I. Métaxas) à 1974 (chute de la dictature des colonels).

5. Rappelons, pour mémoire, qu'à la suite des accords de Zurich en 1959, Chypre devient une République indépendante (1960). 1965 voit l'ouverture démocratique avec les succès électoraux de l'Union du Centre, dirigée par Georges Papandréou, et de l'EDA (Union de la Gauche démocratique).

6. Il faudrait parler en fait de « roméité " pour suivre l'intuition de Y. Ritsos qui, dans son texte

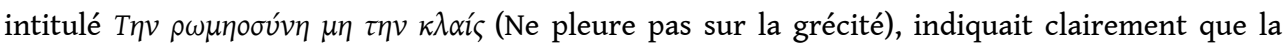
notion d'hellénisme, proposée par les occidentaux, ne pouvait représenter adéquatement une réalité culturelle dont le centre géographique a été l'Anatolie pendant plus d'un millénaire.

7. Il s'agit, précisons-le, du christianisme orthodoxe qui relève des Patriarcats traditionnels de Constantinople, Antioche, Jérusalem et Alexandrie. Cette tradition s'est opposée durant des siècles à l'Église syrienne, dite Jacobite, puis à l'Église apostolique arménienne.

8. Ces chansons pontiques sont organisées en distiques et, comme c'est la règle, leur musique correspond aux rythmes de danses telles que le omal, le kotsari, le kotsankél, etc.

9. Ce qu'il conviendrait d'appeler la roméité, en se référant à l'ethnonyme roméos par lequel les Pontiques se désignaient, ainsi qu'à la réalité historique des millets régionales où se différencient les groupes musulmans, les Grecs orthodoxes et les Arméniens.

10. Les Crétois consommeraient des champignons. Mais l'on doit attribuer certains traits de la culture crétoise à l'influence durable des Vénitiens. Le champignon de Paris en conserve a fait son apparition dans la diète des populations influencées par l'Europe.

11. Le folklore pontique est très homogène, mais il $\mathrm{y} a$, bien sûr, des différences locales. En ce qui concerne les chants, les Pontiques musulmans n'ont plus de chansons en dialecte; ils chantent en turc local.

12. En Grèce, depuis la dictature de Métaxas (1936) et après la guerre civile (1950), les populations rurales ont été désarmées et le port d'arme est contrôlé de façon très restrictive. Le costume masculin, de type caucasien, implique des armes de poing, mais celles-ci ne sont, aujourd'hui encore, que de grossières imitations. Par exemple, la fameuse «danse des glaives " s'exécute avec des substituts de bois.

13. En fait, il y a au moins deux dialectes différents : 1) celui de la région de Tonya, qui est une variété proche de celle de Matsuka ou de la Haldhia septentrionale ; 2) celui de Of, qui reste à ce jour peu décrit. Les pontiques de Grèce sont peu conscients de cette situation dialectale.

14. Les Pontiques de l'ex-URSS constituent une véritable diaspora et, de ce fait, leur rapport au territoire imaginaire est très différent. Pour nombre d'entre eux, c'est déjà le Caucase qui représente un lieu d'autochtonie. La référence à la Turquie originelle existe, mais la forme d'un «ailleurs » totalement indifférencié.

\section{RÉSUMÉS}

Les Gréco-Pontiques de la Turquie orientale ont été installés en Grèce à la suite du Traité de Lausanne (1923). Depuis lors, la «Patrie perdue» a constitué un élément central du cadre 
imaginaire où se réalisent les identifications de la ponticité contemporaine.

Après la guerre civile, le tourisme de pèlerinage en Turquie est devenu, dans les années 60 , de plus en plus facile pour les Grecs. Le retour au territoire d'origine a pour conséquence de modifier le lien existant entre l'imaginaire et la réalité actuelle que tout un chacun peut observer. L'étude de ce processus souligne, entre autres, le rôle que les musulmans ponticophones jouent dans l'élaboration de représentations nouvelles.

The Christian orthodox populations of the Back Sea area, or Pontos, were transfered in Greece according to the exchange agreement of the Lausanne Treaty (1923). Since this date, the Iost country (Patridha) became a sacred issue which works as a basic imaginary component of grecopontic identity inside the remembrance process acting into the family and group history.

After the Greek civil war, the pilgrimage travels became easier and more frequent for the Pontics of Greece. The paper deals with the consequences of the "Tour to Turkey" which evidently modified the link between the lost country of imaginary dwellings and the reality that can be looked at by everybody today. We underline the part that the Pontic speaking moslems play in the process of mental evolution.

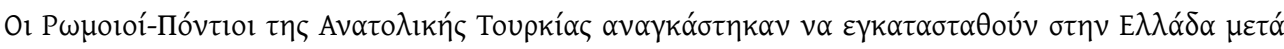

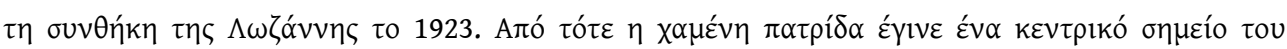

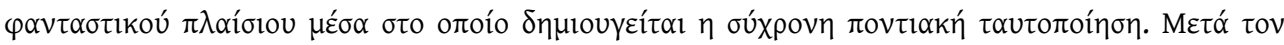

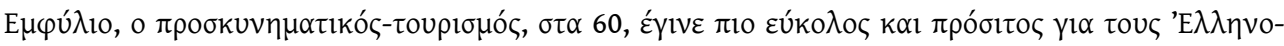

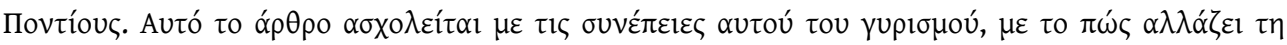

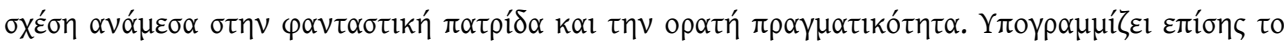

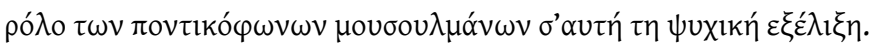

\section{INDEX}

Index géographique : Grèce, Pont, Sumela, Turquie motsclesmk АџИЛАК

motsclestr Hac, Pontus, Pontuslukuk, Sümela

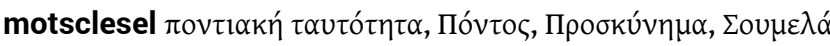

Mots-clés : Adygues, pèlerinage, ponticité, Chalcédonien, Laze, Panaghia Sumela, Pont/

Pontique, Ponticité

Thèmes : Anthropologie, Histoire

Keywords : Greece, Turkey, Pontus, pilgrimage, Sumela, Anthropology

Index chronologique : vingtième siècle 